1 Common vampire bat contact calls attract past food-sharing partners

2 Gerald G. Carter ${ }^{1}$

3 Gerald S. Wilkinson ${ }^{2}$

4 1. Smithsonian Tropical Research Institute, Gamboa, Panama

5 2. Department of Biology, University of Maryland, USA

6

20 Correspondence: Gerald Carter, gerry@ socialbat.org, Smithsonian Tropical Research

21 Institute, Attn: Gerry Carter (Rachel Page Lab, Gamboa), P.O. Box 0843-00153,

22 Panama, Republic of Panama

23 (C) 2016. This manuscript version is made available under the Elsevier user license

24 http://www.elsevier.com/open-access/userlicense/1.0/ 
25 Animals living with kin and non-kin should make social decisions based on the

26 consequences for both direct and indirect fitness. Common vampire bats invest in stable

27 cooperative relationships that benefit both components of inclusive fitness. To

28 disentangle these two factors, we conducted two types of playback trials in a captive

29 group of familiar common vampire bats (Desmodus rotundus) with mixed kinship. We

30 presented each subject with two speakers, playing contact calls from two different

31 familiar conspecifics. In past donor trials, 15 vampire bats chose between calls recorded

32 from either a frequent or rare donor, but both callers were related to a similar degree with

33 the subject. In kinship trials, 31 vampire bats chose between calls recorded from either a

34 close or distant relative, but both callers were matched for food-sharing history with the

35 subject. We found that vampire bats were attracted to contact calls of frequent donors

36 when controlling for kinship, but not to calls of kin when controlling for food-sharing

37 history. Responses and acoustic analyses suggested that bats recognized individuals from

38 vocalizations. These results corroborate past findings that prior food sharing can

39 overshadow relatedness in determining the social decisions of vampire bats. Common

40 vampire bat contact calls allow food-sharing partners to recognize and find each other at

41 a distance, which likely enables them to switch roosts together in the wild.

42

43 Keywords: bats, cooperation, communication, food sharing, individual recognition, kin

44 recognition, social calls, vampire bats, vocal recognition, vocalisations 
Several social birds and mammals_-including some corvids, primates, carnivores,

47 cetaceans, and bats-form individualized and enduring social relationships that involve

48 multiple forms of cooperation (Brent, Chang, Gariépy, \& Platt, 2014; Carter, 2014;

49 Seyfarth \& Cheney, 2012). These cooperative social bonds can provide indirect and

50 direct fitness benefits, so animals should invest in them based on kinship cues (Hamilton,

51 1964), past experiences of reciprocal cooperation (Trivers, 1971), or both (Wilkinson,

52 1984). However, kinship and reciprocal help are often correlated and confounded. An

53 understanding of why animals invest in cooperative relationships can therefore require

54 teasing apart their relative importance (Carter \& Wilkinson, 2013a; Schino \& Aureli, 55 2010).

56 Among adult common vampire bats (Desmodus rotundus), enduring reciprocal

57 relationships appear to stabilize regurgitated food sharing (Carter \& Wilkinson, 2013a,

58 2013b; Wilkinson, 1984; 1985a; 1986). Food sharing has inclusive fitness consequences

59 because vampire bats often fail to feed and can starve to death after missing only 2-3

60 nightly meals of blood (Wilkinson, 1984; 1988). Food-sharing rates among dyads in a

61 social network are predicted by several correlated factors: kinship, association,

62 allogrooming, and reciprocal sharing. The relative roles of kinship versus social

63 experience have been assessed by Monte Carlo simulation (Wilkinson, 1988), and by

64 testing predictors of food sharing among bats observed under natural conditions

65 (Wilkinson, 1984), among unrelated bats with different levels of association (Wilkinson,

66 1984), and among familiar bats with different levels of relatedness (Carter \& Wilkinson,

67 2013a). These tests have shown that past experiences of food sharing or social grooming

68 can overshadow kinship for predicting food donation rates among adults (Carter \& 
69 Wilkinson 2013b). The hypothesis that food sharing provides reciprocal benefits is

70 further supported by the observation that vampire bats that feed more non-kin maintain a

71 larger and more robust network of donors (Carter \& Wilkinson, 2015). However, the

72 most common and crucial context for food sharing involves mothers feeding their

73 dependent offspring, suggesting that regurgitated food sharing may have evolutionary

74 origins in maternal care.

75 Another possibly neotonous social behaviour in vampire bats is contact calling. In

76 all bat species, pups produce isolation calls that allow mothers to recognize and retrieve

77 them (Balcombe, 1990; Bohn, Moss, \& Wilkinson, 2006; Carter \& Wilkinson, 2013c;

78 Gould, 1971; Gould, Woolf, \& Turner, 1973; Scherrer \& Wilkinson, 1993; Wilkinson,

79 2003). In all three vampire bat species, contact calls are also produced by isolated adults

80 (Carter, Logsdon, Arnold, Menchaca, \& Medellin, 2012). These calls can be recorded by

81 isolating an individual for a prolonged period, they are longer and lower-frequency than

82 echolocation pulses and so travel farther distances, and they vary substantially in acoustic

83 structure both among and within individuals (Fig. 1). Contact calls appear to allow bats to

84 find and recognize familiar groupmates. In white-winged vampire bats (Diaemus youngi),

85 vocal discrimination was evident because vocal responses occurred more often when

86 playbacks of different contact calls shifted to a novel caller (Carter, Skowronski, Faure,

$87 \&$ Fenton, 2008), and also when 2-3 isolated group-mates called from swapped spatial

88 positions rather than from each of their original locations (Carter, Fenton, \& Faure,

89 2009). Vocal discrimination has not yet been experimentally demonstrated among adult

90 common vampire bats.

91 To determine the social factors that influence responses to contact calls in 
92 common vampire bats, we conducted two kinds of playback trials. In past donor trials, we

93 compared the attraction of subjects to calls recorded from two individuals matched by

94 kinship but differing in their history of donating food to the subject. We predicted that the

95 isolated subjects would be attracted to calls of the more frequent donor.

96 In kinship trials, we compared the attraction of subjects to calls recorded from

97 bats matched in food-sharing history but contrasting in kinship with the subject. Since we

98 previously found that reciprocal sharing is eight times more important than kinship for

99 predicting donation rates (Carter \& Wilkinson 2013a), we predicted that responses during

100 kinship trials would show a weaker effect size compared with past donor trials. In sum,

101 we predicted that isolated vampire bats would seek out past food donors more strongly

102 than relatives.

103

104 METHODS

105 Subjects

106 Subjects were 31 common vampire bats (Desmodus rotundus), from five

107 matrilines and three zoo populations, housed and cared for during the study by the

108 Organization of Bat Conservation at the Cranbrook Institute of Science (Bloomfield

109 Hills, Michigan) under permits from the USDA (34-C-0117) and the US Fish and

110 Wildlife Service (MB003342-0). Unrelated bats from the different populations were

111 introduced as adults. Bats were housed at 25-28 degrees Celsius with $>33 \%$ humidity on

112 a $12 \mathrm{~h} \mathrm{light/dim} \mathrm{light} \mathrm{cycle} \mathrm{in} \mathrm{a} 3 \times 1.5 \times 2 \mathrm{~m}$ cage with a soft paper bedding floor that

113 allowed flight and free association among cagemates. Bats were fed on physically or

114 chemically defibrinated bovine blood that was available ad libitum and changed twice 
115 daily. All procedures with animals adhered to the Animal Behavior Society Guidelines

116 for the Use of Animals in Research and were approved by the University of Maryland

117 Institutional Animal Care and Use Committee (Protocol R-10-63).

\section{Social data}

119 Measures of food sharing history were derived from a food-sharing network

120 involving 355 trial-donations and more than 1250 regurgitation observations made from

1212010 - 2014. To quantify food sharing history we used a food sharing index from A to B,

122 which we calculated as the natural $\log$ of $(\mathrm{X}+1)$ where $\mathrm{X}$ is the food sharing rate from $\mathrm{A}$

123 to B (s of mouth-licking per $1 \mathrm{~h}$ of experimental trial). To calculate relatedness, we

124 genotyped 19 microsatellite markers (Carter \& Wilkinson, 2015) and used the Wang

125 estimator (Wang, 2002) calculated in the R package related (Pew, Muir, Wang, \&

126 Frasier, 2015). We defined kinship by substituting zeros for all negative relatedness

127 values and replacing relatedness values of known maternal kin with the appropriate

128 pedigree kinship values (e.g. mother $=0.5$, grandmother $=0.25$ ). Findings remained the

129 same whether we used kinship or relatedness.

$130 \quad$ Playback stimuli

131 Isolated vampire bats produce multi-harmonic contact calls that are variable

132 between and within individual bats (Fig. 1; Carter et al., 2012). We recorded contact calls

133 by placing an adult individual in a small soft mesh butterfly cage at a distance of 10-30

$134 \mathrm{~cm}$ to a CM16 ultrasound condenser microphone (frequency range 10-200 kHz, Avisoft

135 Bioacoustics, Berlin, Germany) inside an acoustic recording booth (plastic bin lined with

136 acoustic foam) for 1-24 h. Due to these ideal recording conditions (e.g. ultrasonic

137 microphone close to the bat's mouth, low gain, semi-anechoic chamber), the noise level 
138 was low ( $<0.5 \%$ amplitude). We selected the first $17-30$ contact calls that were between

139 10-99\% amplitude (100\% amplitude signals are clipped) to use in playbacks. Sounds

140 were digitized with 16-bit resolution at a sampling rate of $250 \mathrm{kHz}$ through an Avisoft

141 Ultrasoundgate 116 to a laptop running the program Avisoft Recorder.

142 We created 19 playback sequences from 19 different vampire bats. For each

143 sequence, we used Batsound Pro (Pettersson Elektronik AB, Sweden) to space apart

144 contact calls at random inter-call intervals varying from 1-3 s, and then adjusted intervals

145 so that 30 calls would fit in a $60 \mathrm{~s}$ sequence. To remove sounds below $1 \mathrm{kHz}$, we used a

146 high-pass Butterworth filter (filter order=2). Each 60 s playback sequence was presented

147 and repeated four times in a trial through one of two Avisoft UltraSoundGate Player BL

148 Pro units (henceforth "speakers"), which each contain an integrated digital-to-analogue

149 converter, amplifier, and ultrasonic speaker driver (see www.ultrasoundgate.com for

150 frequency response curves). To equalize the intensity of the two speaker signals, we used

151 the Avisoft recording system described above to simultaneously monitor the amplitudes

152 of sounds from the two speakers playing the same contact call repeatedly at equal

153 distances to the microphone, and we then adjusted the speaker gains until the signal

154 amplitudes matched.

\section{Playback experiment}

156 Trials took place in darkness and were filmed with an infrared spotlight (IRlamp6,

157 Wildlife Engineering, USA) and a Sony Nightshot DCR-SR85 camcorder. In each trial, a

158 subject bat was placed in a $76 \times 76 \mathrm{~cm}$ plastic mesh experimental 'maze' with five arms

159 that allowed the subject bat in the center of the maze to move forwards, backwards, left,

160 right, or upwards (Figure 2). The bat was placed in the arm farthest from the camera, 
161 equidistant to the two speakers. After moving forwards, it could enter the arms to the left

162 or right —each option leading towards a speaker simultaneously playing calls from either

163 a control bat or a test bat. The bat could also move straight backwards or forwards away

164 from either speaker, or climb upwards, which bats typically do as an escape

165 response. These options allowed us to remove cases where bats were not motivated to

166 move toward either speaker.

167 Playback trials were of one of two types: past donor trials and kinship trials. In

168 past donor trials, a subject with a history of food sharing ( $\mathrm{N}=12$ females, 3 males) was

169 presented with a choice between approaching contact calls from a frequent food donor

170 (test bat) and an infrequent or non-donor (control bat). Test and control bats differed in

171 their food donation rates to the subject, but were matched by age and kinship (Table 1).

172 In kinship trials, a subject ( $\mathrm{N}=12$ females, 19 males) was presented with a choice

173 between contact calls from a close relative (test bat) and a distant or non-relative (control

174 bat). Test and control bats differed in their pairwise kinship with subject, but were closely

175 matched wherever possible by age and past food-sharing experience (Table 1).

176 Each trial lasted $10 \mathrm{~min}$. If the bat did not make a choice within the first trial, the

177 trial was discarded and a new trial began. Test stimuli were presented on the left or right

178 randomly. The maze was rotated $90^{\circ}$ clockwise between each trial. We presented the two

179 trial types on a random schedule to remove order effects. Each subject experienced a

180 unique playback combination of test and control bat except for one test-control

181 combination that was presented to two different male subjects in the kinship trials;

182 however, removing either one of these observations does not change our conclusions.

183 Playback response analysis 
185 playback treatments and design. The blind observers recorded (1) the number of seconds

186 that bats spent in either the left or right arm of the maze and (2) the latency in seconds

187 from the start of a trial until the bat first entered either the right or left arm. If the bat

188 never entered the arm, latency was scored as $600 \mathrm{~s}$. Response data were non-normal, so

189 we calculated p-values using bootstrapping. Using the R package boot, we resampled the

190 mean differences (test-control) for both the subject's time in each arm and subject's

191 latency to enter them, and calculated the one-sided p-value as the proportion of the 5000

192 sample means that exceeded the observed mean. We calculated $95 \%$ confidence intervals

193 (CI) using the BCa method (Puth, Neuhäuser, \& Ruxton, 2015). Like all inferences,

194 resampling tests can be problematic when a sample is too small to properly capture the

195 variation of the population. For inferences based on small sample sizes $(<20)$, we

196 therefore also present more conservative p-values calculated using a non-parametric

197 Wilcoxon signed ranks test. All response data are available on FigShare (Carter 2015).

198 Acoustic analysis of playback stimuli

199 We performed acoustic analyses of the playback stimuli to help interpret our

200 behavioural results. A bias towards frequent donors or kin could indicate vocal

201 recognition. Alternatively, without vocal recognition, an attraction towards kin or

202 frequent donors could occur if kin or frequent donors sound more alike and subjects were

203 attracted to more similar-sounding group-mates. We therefore tested if the pairwise

204 relatedness or food-sharing index between the callers predicted the acoustic distance

205 between all possible pairs of 19 playbacks. To measure acoustic distance, we used

206 automated measures in Avisoft SASLab Pro to extract syllable duration; distance to 
207 maximum amplitude, and peak frequencies at the call's start, end, and maximum

208 amplitude (FFT 512, Frame size $=100 \%$, Hann window), then entered these variables into

209 a linear discriminant function analyses in R. We defined 'acoustic distance' as the natural

$210 \log$ of the Mahalanobis distance between the centroids for each playback.

211 We also tested the null assumptions that the absolute difference in either acoustic

212 distance or mean peak-to-peak amplitude between the test and control playbacks did not

213 differ by (1) treatment, (2) time the subject spent in the test arm, or (3) latency for the

214 subject to enter the test arm. To test these assumptions, we used permutation tests applied

215 to general linear models using lmPerm package (Wheeler, 2010) in R. Such an

216 unconstrained randomisation approach can have higher power than a Mantel test

217 (Legendre \& Fortin, 2010) for detecting any potential effects of acoustic distance or

218 amplitude difference on bat response. We calculated percentage difference in relative

219 intensity between test and control playbacks by subtracting the mean amplitude of the

220 control playback from that of the test playback, and then dividing by the highest recorded

221 amplitude. Finally, to confirm individuality of the playbacks, we performed jackknifed

222 linear discriminant function analysis in R.

224 RESULTS

225 Vampire bats placed in the test arena typically walked and echolocated towards

226 one speaker and then the other, alternating between them. In past donor trials, subjects

227 were more attracted to calls of more frequent donors compared to less frequent donors.

228 They spent $51 \mathrm{~s}$ longer in the test arm than in the control arm (95\% CI=12-91 s; $\mathrm{n}=15$,

229 permutation $\mathrm{p}=0.0014$; Wilcoxon $\mathrm{S}=44$, one-sided $\mathrm{p}=0.005$, Figure $3 \mathrm{~A}$ ). Attraction to the 
230 frequent donor was still evident even when only using the 9 trials where the test bat was

231 unrelated to the subject (test kinship $<0.02$; mean time in test arm $=57 \mathrm{~s}, 95 \% \mathrm{CI}=16$

$232151 \mathrm{~s}$, permutation $\mathrm{p}=0.007$; Wilcoxon $\mathrm{S}=15.0$, one-sided $\mathrm{p}=0.039)$. Subjects also entered

233 the test arm $184 \mathrm{~s}$ sooner $(95 \% \mathrm{CI}=42-331 \mathrm{~s}$; permutation $\mathrm{p}=0.0066$; Wilcoxon $\mathrm{S}=34.5$,

234 one-sided $\mathrm{p}=0.025$, Figure $3 \mathrm{~B}$ ).

235 In contrast, subjects in kinship trials did not spend longer in the test arm with calls

236 from kin compared to the control arm with calls from non-kin (95\% CI of difference: -

$23713-33 \mathrm{~s} ; \mathrm{n}=31, \mathrm{p}=0.192$, Figure $3 \mathrm{~A}$ ). The latency to enter the test arm also did not differ

$238(95 \% \mathrm{CI}=-47-114 \mathrm{~s}$, Figure 3B). Subjects were not attracted to the eight related non-

239 donors (95\% CI $=-58-51 \mathrm{~s})$, and we detected no kinship preference in either male (95\%

$240 \mathrm{CI}=-28-37 \mathrm{~s})$ or female subjects $(95 \% \mathrm{CI}=-18-43 \mathrm{~s})$, and no bias for adults towards

241 their mothers $(\mathrm{n}=19,95 \% \mathrm{CI}=-37-23 \mathrm{~s})$. However, five of the six mothers spent more

242 time near the calls of their adult offspring than to calls from a control bat $(\mathrm{n}=6,95 \% \mathrm{CI}=$

$2437-86 \mathrm{~s}$, permutation $\mathrm{p}=0.0074$; Wilcox on $\mathrm{S}=8.5$, one-sided $\mathrm{p}=0.047$ ).

244 The absolute difference in relative intensity between test and control playbacks

245 did not differ from zero $(95 \% \mathrm{CI}=-2-4 \%)$, and neither relative intensity difference nor

246 acoustic distance between the test and control playbacks correlated with behavioural

247 response times or test type (Table 2). Natural-log-transformed centroid distance also did

248 not correlate with either the relatedness $\left(n=171, R^{2}=0.005, p=0.36\right)$ or food-sharing

$249\left(\mathrm{n}=88, \mathrm{R}^{2}=0.013, \mathrm{p}=0.28\right)$ between the two bats used for the playbacks. Although neither

250 kinship nor food-sharing history predicted acoustic similarity between playbacks, correct

251 assignment rates of playback calls to bat was greater than chance (5\% with random 
252 chance vs. $20 \%$ of training set or $18 \%$ cross-validated; Wilks' Lambda $=0.389, \chi^{2}=$

$253688.43, \mathrm{df}=90, \mathrm{p}<0.001)$.

\section{DISCUSSION}

255 The common vampire bats in this study were attracted to the contact calls of

256 frequent donors, even when controlling for kinship. In contrast, they were not attracted to

257 contact calls of relatives when controlling for food-sharing history. The exception was

258 that mothers were attracted to calls of their adult male offspring. We conducted more

259 kinship trials than past donor trials, so a lack of power is unlikely to explain the

260 difference in responses between the trial types. Past findings in this same captive group

261 found that reciprocal sharing and social grooming can overshadow kinship for

262 determining food-sharing decisions (Carter \& Wilkinson, 2013b), and our results here

263 suggest that the same is true for decisions about responding to contact calls.

264 In female greater spear-nosed bats Phyllostomus hastatus, social affiliation leads

265 to convergence in contact call structure, and females are attracted to group-specific

266 contact calls without individual vocal recognition (Boughman \& Wilkinson, 1998). In

267 contrast, we found no evidence that attraction to frequent donors occurred because

268 frequent donors sound more alike and that subjects were merely attracted to more similar-

269 sounding group-mates. Instead, attraction to frequent donors is consistent with individual

270 vocal recognition. Playback responses are not easily explained by alternative

271 explanations. Playbacks in the kinship trials were not acoustically more similar than those

272 in past donor trials, and we did not detect weaker behavioural responses when the test and

273 control bats were more acoustically similar in frequency or intensity. We also did not find

274 evidence indicating that playbacks from related bats or frequent donors sounded more 
275 similar to each other, suggesting that responses were not based on self-phenotype 276 matching of calls.

277 Measures of only a few acoustic parameters - duration, distance to maximum

278 amplitude and peak frequency at three points - provided better than chance classification

279 of call to individual, and bats are likely using a much richer set of acoustic information in

280 these richly harmonic calls, such as formant-like spectral features shaped by the vocal

281 tract (Yovel, Melcon, Franz, Denzinger, \& Schnitzler, 2009). Rather than merely being

282 attracted to certain call characteristics, the acoustic and behavioural results suggest that

283 bats were matching calls to a memory of a specific groupmate, and basing their decisions

284 on caller identity. This ability would not be surprising given the experimental evidence

285 that white-winged vampire bats (Diaemus youngi) can discriminate among familiar

286 conspecifics using similar contact calls (Carter et al., 2009; Carter et al., 2008), greater

287 spear-nosed bats (Phyllostomus hastatus) can recognize individual pups using isolation

288 calls (Bohn, Wilkinson, \& Moss, 2007), and greater mouse-eared bats (Myotis myotis)

289 can recognize individuals using voice characteristics of echolocation calls (Yovel et al.,

290 2009), which function primarily for biosonar.

291 With regards to kin discrimination, the results here are consistent with two

292 interpretations. Either the bats were not able to use calls to recognize kinship, or any

293 perceived kinship was overshadowed by social experience. This latter hypothesis is

294 consistent with prior evidence on social decision-making by vampire bats. Free-ranging

295 female vampire bats both positively assort and share food with close kin, and this pattern

296 is unlikely to result from simple mechanisms for kin-biased association, such as spatial

297 location or familiarity (Penn \& Frommen, 2010), because female common vampire bats 
298 develop multi-year stable associations with kin and non-kin even when frequently

299 switching roosts (Tschapka \& Wilkinson, 1999; Wilkinson, 1984; Wilkinson, 1985a,

300 1985b). The mechanisms of kin discrimination in vampire bats remain unclear. Given the

301 results presented here, a parsimonious explanation is that vampire bats learn the calls of

302 maternal kin based on early life experiences (prior association) and could not recognize

303 or favour unfamiliar kin (phenotype matching). However, there is growing recognition

304 that social vertebrates may have multiple forms of kin discrimination, including both

305 prior association and phenotype matching (Breed, 2014; Cornwallis, West, \& Griffin,

306 2009; Green et al., 2015; Mateo, 2010; Mateo \& Hauber, 2015; Mcdonald \& Wright,

307 2011; Pfefferle, Kazem, Brockhausen, Ruiz-Lambides, \& Widdig, 2014). Different kin

308 recognition mechanisms may be used in different contexts (e.g. inbreeding avoidance vs.

309 nepotism) and might interact with past experience in various ways to influence social

310 decisions (Breed, 2014; Daniel \& Rodd, 2015; Delton, Krasnow, Cosmides, \& Tooby;

311 Mateo, 2010; Mateo \& Hauber, 2015; Mcdonald \& Wright, 2011; Penn \& Frommen,

312 2010; Zöttl, Heg, Chervet, \& Taborsky, 2013). Like most mammals, bats appear to use

313 olfaction for social recognition at close range (Bloss, Acree, Bloss, Hood, \& Kunz, 2002;

314 De Fanis \& Jones, 1995; Englert \& Greene, 2009; Gustin \& McCracken, 1987; Safi \&

315 Kerth, 2003), but a system of kin recognition based on acoustic phenotype matching

316 (Kessler, Scheumann, Nash, \& Zimmermann, 2012; Levréro et al., 2015; Pfefferle, Ruiz-

317 Lambides, \& Widdig, 2014) would allow for recognition of unfamiliar or paternal

318 relatives at a distance, which would be necessary for co-roosting preferentially with

319 unfamiliar kin over unfamiliar non-kin. Analysis of call variation using better feature 
320 extraction across a larger sample of calls and bats is therefore needed to determine if

321 there are subtle kinship cues that can be used to discriminate unfamiliar (paternal) kin.

322 Common vampire bats are likely to use contact calls in at least two cooperative

323 contexts. First, common vampires, like many forest-dwelling bats, possess 'fission-

324 fusion' social dynamics where stable relationships are maintained despite frequent re-

325 grouping and roost switching (Aureli et al., 2008; Kerth, Perony, \& Schweitzer, 2011;

326 Popa-Lisseanu, Bontadina, Mora, \& IbÁñez, 2008; Willis \& Brigham, 2004). In Costa

327 Rica, particular females often ended up in the same roost despite frequently moving

328 among 1-6 hollow tree roosts (Wilkinson, 1985a). Given the importance of acoustic

329 communication for bats, and the time and energy it would take to visit multiple trees to

330 smell roost-mates in each, it seems most likely that vampire bats use vocalizations to

331 coordinate these co-roosting associations, as has been found in other bat species (Arnold

332 \& Wilkinson, 2011; Chaverri, Gillam, \& Kunz, 2012; Kerth \& Reckardt, 2003). Second,

333 contact calls might be used within the roost. Food donors often initiate and approach

334 hungry bats to feed them (Carter \& Wilkinson, 2013b). In fact, they will sometimes fly to

335 trapped hungry partners, and even feed them through cage bars (Carter, 2016), suggesting

336 that vampire bats can recognize a needy partner from a distance.

\section{ACKNOWLEDGEMENTS}

339 This study was made possible by collaboration with the Organization for Bat

340 Conservation, with help from R. Mies, A. Felk, N. Siekert, J. Fabian, L. Dehn, and K.

341 Vowles. The experimental maze was built by K. Sullenger. The work was supported by

342 student research grants from the Animal Behavior Society and the American Society of 
343 Mammalogists, and a Doctoral Dissertation Improvement Grant from the National

344 Science Foundation (\#1311336). We thank Brock Fenton, Ken Yasukawa, and an

345 anonymous reviewer for suggestions that improved the manuscript.

347 REFERENCES

349 Arnold, B. D., \& Wilkinson, G. S. (2011). Individual specific contact calls of pallid bats 350 (Antrozous pallidus) attract conspecifics at roosting sites. Behavioral Ecology and $351 \quad$ Sociobiology, 65, 1581-1593.

352 Aureli, F., Schaffner, C. M., Boesch, C., Bearder, S. K., Call, J., Chapman, C. A. et al. 353 (2008). Fission- fusion dynamics. Current Anthropology, 49, 627-654.

354 Balcombe, J. P. (1990). Vocal recognition of pups by mother Mexican free-tailed bats, 355 Tadarida brasiliensis mexicana. Animal Behaviour, 39, 960-966.

356 Bloss, J., Acree, T., Bloss, J., Hood, W., \& Kunz, T. (2002). Potential use of chemical cues for colony-mate recognition in the big brown bat, Eptesicus fuscus. Journal

359 Bohn, K. M., Moss, C. F., \& Wilkinson, G. S. (2006). Correlated evolution between 360 hearing sensitivity and social calls in bats. Biology Letter, 2, 561-564.

361 Bohn, K. M., Wilkinson, G. S., \& Moss, C. F. (2007). Discrimination of infant isolation 362 calls by female greater spear-nosed bats, Phyllostomus hastatus. Animal 363 Behaviour, 73, 423-432.

364 Boughman, J. W., \& Wilkinson, G. S. (1998). Greater spear-nosed bats discriminate 365 group mates by vocalizations. Animal Behaviour, 55, 1717-1732. 
366 Breed, M. D. (2014). Kin and nestmate recognition: the influence of W. D. Hamilton on 36750 years of research. Animal Behaviour, 92, 271-279. doi: 10.1016/j.anbehav.2014.02.030

369 Brent, L. J., Chang, S. W., Gariépy, J. F., \& Platt, M. L. (2014). The neuroethology of 370 friendship. Annals of the New York Academy of Sciences, 1316, 1-17.

371 Carter, G. G. (2014). The reciprocity controversy. Animal Behavior and Cognition, 1, 372 368-386. doi: 0.12966/abc.08.11.2014

373 Carter, G. (2016): Footage of regurgitated food sharing among adult common vampire $374 \quad$ bats. Figshare. https://dx.doi.org/10.6084/m9.figshare.2067417.v1

375 Carter, G. G., Fenton, M. B., \& Faure, P. (2009). White-winged vampire bats (Diaemus 376 youngi) exchange contact calls. Canadian Journal of Zoology, 87, 604-608.

377 Carter, G. G., Logsdon, R., Arnold, B., Menchaca, A., \& Medellin, R. A. (2012). Adult 378 vampire bats produce contact calls when isolated: acoustic variation between $379 \quad$ species, colonies, and individuals. PLOS ONE, 7, e38791.

380 Carter, G. G., Skowronski, M., Faure, P., \& Fenton, M. B. (2008). Antiphonal calling

381 allows individual discrimination in white-winged vampire bats. Animal 382 Behaviour, 76, 1343-1355.

383 Carter, G. G., \& Wilkinson, G. S. (2013a). Food sharing in vampire bats: reciprocal help 384 predicts donations more than relatedness or harassment. Proceedings of the Royal Society of London Series B, 280, 20122573. doi: 10.1098/rspb.2012.2573

386 Carter, G. G., \& Wilkinson, G. S. (2013b). Does food sharing in vampire bats 387 demonstrate reciprocity? Communicative and Integrative Biology, 6, e25783. doi:

$388 \quad 10.4161 / \mathrm{cib} .25783$ 
389 Carter, G. G., \& Wilkinson, G. S. (2013c). Cooperation and conflict in the social lives of $390 \quad$ bats. In R. Adams \& S. Pedersen (Eds.), Bat Evolution, Ecology, and 391 Conservation (pp. 225-242). New York: Springer Science Press.

392 Carter, G. G., \& Wilkinson, G. S. (2015). Social benefits of non-kin food sharing by 393 female vampire bats. Proceedings of the Royal Society of London Series B, 282, 394 20152524-20152524. doi: 10.1098/rspb.2015.2524

395 Chaverri, G., Gillam, E. H., \& Kunz, T. H. (2012). A call-and-response system facilitates 396 group cohesion among disc-winged bats. Behavioral Ecology, 24, 481-487. 397 doi:10.1093/beheco/ars188

398 Cornwallis, C., West, S., \& Griffin, A. (2009). Routes to indirect fitness in cooperatively 399 breeding vertebrates: kin discrimination and limited dispersal. Journal of 400 Evolutionary Biology, 22, 2445-2457. doi:10.1111/j.1420-9101.2009.01853.x.

401 Daniel, M. J., \& Rodd, F. H. (2015). Female guppies can recognize kin but only avoid 402 incest when previously mated. Behavioral Ecology, 27, 55-61.

403 doi: $10.1093 /$ beheco/arv122

404 De Fanis, E., \& Jones, G. (1995). The role of odour in the discrimination of conspecifics 405 by pipistrelle bats. Animal Behaviour, 49, 835-839.

406 Delton, A. W., Krasnow, M. M., Cosmides, L., \& Tooby, J. Evolution of direct 407 reciprocity under uncertainty can explain human generosity in one-shot 408 encounters. Proceedings of the National Academy of Sciences USA, 108, 13335$409 \quad 13340$. 
410 Englert, A. C., \& Greene, M. J. (2009). Chemically-mediated roostmate recognition and roost selection by Brazilian free-tailed bats (Tadarida brasiliensis). PLOS ONE, 4(11), e7781.

413 Gould, E. (1971). Studies of maternal-infant communication and development of vocalizations in the bats Myotis and Eptesicus. Communications in Behavioral Biology, 5, 263-313.

416 Gould, E., Woolf, N., \& Turner, D. C. (1973). Double-note communication calls in bats: 417 occurrence in three families. Journal of Mammalogy, 54, 998-1001.

418 Green, Jonathan P., Holmes, Andrew M., Davidson, Amanda J., Paterson, S., Stockley, P., Beynon, Robert J., \& Hurst, Jane L. (2015). The genetic basis of kin recognition in a cooperatively breeding mammal. Current Biology. 25, 26312641. doi: 10.1016/j.cub.2015.08.045

424 Hamilton, W. D. (1964). The genetical evolution of social behavior. Journal of 425 Theoretical Biology, 7, 1-51.

426 Kerth, G., Perony, N., \& Schweitzer, F. (2011). Bats are able to maintain long-term social 427 relationships despite the high fission-fusion dynamics of their groups. 428 Proceedings of the Royal Society of London Series B, 278, 2761-2767. doi: $10.1098 /$ rspb.2010.2718

430 Kerth, G., \& Reckardt, K. (2003). Information transfer about roosts in female Bechstein's 431 bats: an experimental field study. Proceedings of the Royal Society of London, $432 \quad$ Series B, 270, 511-515. 
433 Kessler, S. E., Scheumann, M., Nash, L. T., \& Zimmermann, E. (2012). Paternal kin recognition in the high frequency/ultrasonic range in a solitary foraging mammal. BMC Ecology, 12, 26. doi:10.1186/1472-6785-12-26

436 Legendre, P., \& Fortin, M. J. (2010). Comparison of the Mantel test and alternative approaches for detecting complex multivariate relationships in the spatial analysis of genetic data. Molecular Ecology Resources, 10, 831-844. doi: 10.1111/j.1755-

440 Levréro, F., Carrete-Vega, G., Herbert, A., Lawabi, I., Courtiol, A., Willaume, E., . . .

441 Charpentier, M. (2015). Social shaping of voices does not impair phenotype matching of kinship in mandrills. Nature Communications, 6 . doi:10.1038/ncomms8609

444 Mateo, J. M. (2010). Self-referent phenotype matching and long-term maintenance of kin 445 recognition. Animal Behaviour, 80, 929-935. doi:10.1016/j.anbehav.2010.08.019

446 Mateo, J. M., \& Hauber, M. (2015). Perspectives: Hamilton's Legacy: mechanisms of kin 447 recognition in humans. Ethology, 121, 419-427. doi: 10.1111/eth.12358

448 Mcdonald, P. G., \& Wright, J. (2011). Bell miner provisioning calls are more similar among relatives and are used by helpers at the nest to bias their effort towards kin.

$450 \quad$ Proceedings of the Royal Society of London Series B. 278, 3403-3411.

451 Penn, D. J., \& Frommen, J. G. (2010). Kin recognition: an overview of conceptual issues, 452 mechanisms and evolutionary theory. In P. Kappeler (Ed.) Animal Behaviour:

453 Evolution and Mechanisms (pp. 55-85). Berlin Heidelberg: Springer. 
454 Pew, J., Muir, P. H., Wang, J., \& Frasier, T. R. (2015). related: an R package for

455

456

457

458

459

460

461 Pfefferle, D., Ruiz-Lambides, A. V., \& Widdig, A. (2014). Female rhesus macaques

462

463

464

465

466

467

468

469

470

471

472

473

474 Scherrer, J. A., \& Wilkinson, G. S. (1993). Evening bat isolation calls provide evidence 475

analysing pairwise relatedness from codominant molecular markers. Molecular Ecology Resources, 15, 557-561.

Pfefferle, D., Kazem, A. J., Brockhausen, R. R., Ruiz-Lambides, A. V., \& Widdig, A. (2014). Monkeys spontaneously discriminate their unfamiliar paternal kin under natural conditions using facial cues. Current Biology, 24, 1806-1810. doi: 10.1016/j.cub.2014.06.058 discriminate unfamiliar paternal sisters in playback experiments: support for acoustic phenotype matching. Proceedings of the Royal Society of London Series $B, 281,20131628$.

Popa-Lisseanu, A. G., Bontadina, F., Mora, O., \& IbÁñez, C. (2008). Highly structured fission-fusion societies in an aerial-hawking, carnivorous bat. Animal Behaviour, $75,471-482$.

Puth, M. T., Neuhäuser, M., \& Ruxton, G. D. (2015). On the variety of methods for calculating confidence intervals by bootstrapping. Journal of Animal Ecology, 84, 892-897.

Safi, K., \& Kerth, G. (2003). Secretions of the interaural gland contain information about individuality and colony membership in the Bechstein's bat. Animal Behaviour, $65,363-369$. for heritable signatures. Animal Behaviour, 46, 847-860. 
476 Schino, G., \& Aureli, F. (2010). The relative roles of kinship and reciprocity in 477 explaining primate altruism. Ecology Letters, 13, 45-50.

478 Seyfarth, R. M., \& Cheney, D. L. (2012). The evolutionary origins of friendship. Annual 479 Review of Psychology, 63, 153-177. doi: 10.1146/annurev-psych-120710-100337

480 Trivers, R. L. (1971). The evolution of reciprocal altruism. Quarterly Review of Biology, $481 \quad 46,35-57$.

482 Tschapka, M., \& Wilkinson, G. S. (1999). Free-ranging vampire bats (Desmodus

Wang, J. (2002). An estimator for pairwise relatedness using molecular markers.

$486 \quad$ Genetics, 160, 1203-1215.

487 Wheeler, R. E. (2010). Permutation tests for linear models in R. Available at http://cran. r-project. org/web/packages/lmPerm/vignettes/lmPerm.pdf [accessed 10 September 2013].

490 Wilkinson, G. S. (1984). Reciprocal food sharing in the vampire bat. Nature, 308, 181$491 \quad 184$.

492 Wilkinson, G. S. (1985a). The social organization of the common vampire bat: I. Pattern and cause of association. Behavioral Ecology and Sociobiology. 17, 111-121

494 Wilkinson, G. S. (1985b). The social organization of the common vampire bat: II. Mating 495 496

497 Wilkinson, G. S. (1986). Social grooming in the common vampire bat, Desmodus 498 rotundus. Animal Behaviour, 34, 1880-1889. 
499 Wilkinson, G. S. (1988). Reciprocal altruism in bats and other mammals. Ethology and $500 \quad$ Sociobiology, $9,85-100$.

501 Wilkinson, G. S. (2003). Social and vocal complexity in bats. In F. B. de Waal \& P. L.

502 Tyack (Eds.), Animal Social Complexity: Intelligence, Culture and Individualized

503 Societies (pp. 322-341). Cambridge, MA: Harvard University Press.

504 Willis, C. K., \& Brigham, R. M. (2004). Roost switching, roost sharing and social

505 cohesion: forest-dwelling big brown bats, Eptesicus fuscus, conform to the

506 fission-fusion model. Animal Behaviour, 68, 495-505.

507 Yovel, Y., Melcon, M. L., Franz, M. O., Denzinger, A., \& Schnitzler, H. U. (2009). The

508 voice of bats: how greater mouse-eared bats recognize individuals based on their

509 echolocation calls. PLOS Computational Biology, 5, e1000400.

$510 \quad$ doi:10.1371/journal.pcbi.1000400

511 Zöttl, M., Heg, D., Chervet, N., \& Taborsky, M. (2013). Kinship reduces alloparental

512 care in cooperative cichlids where helpers pay-to-stay. Nature Communications,

$513 \quad 4,1341$. doi:10.1038/ncomms2344

514

515 FIGURE CAPTIONS

516

517 Figure 1. Example contact calls from common vampire bats. Three calls recorded

518 from an 8-year old female (A), 10-year old female (B), 6-year old male (C), and 2.5-year

519 old female (D); all bats were isolated during recording. See Carter et al. 2012 for

520 descriptions of call structure variation.

521 
522 Figure 2. Playback maze. Not shown to scale. Laptops for running each speaker were

523 located in an adjacent room. The cage and the camcorder with infrared light were

524 elevated using a small table and tripod, respectively. The camera was further from the

525 maze than depicted such that the bat could be seen anywhere in the maze.

526

527 Figure 3. Vampire bats were attracted to contact calls from past donors of similar

528 kinship but not to close kin of similar food-sharing history. Time spent (A), or latency

529 to enter (B), the test arm shown for males (red) and females (blue) when calls from

530 frequent donors (donor test) or close relatives (kinship test) were broadcast from the test

531 arm. Blue shading shows probability density function. Results remained the same with or

532 without the two outliers in Panel A.

533

534 TABLES

535 Table 1. Food sharing and kinship of callers with subject bat

\section{Playback treatment}

Past donor Test bat

Control bat

Test - Control

Kinship

Test bat

Control bat

Test - Control
Food sharing index

Median Range $\mathrm{N}$

$4.9 \quad 2.5-6.2 \quad 15$

$0 \quad 0-5.0 \quad 15$

$4.8 \quad 0.8-6.2 \quad 15$

$5.5 \quad 0-7.4 \quad * 24$

$\begin{array}{llll}0 & 0-4.9 & * 19\end{array}$

$\begin{array}{lll}0 & 0-7.3 & * 18\end{array}$
Kinship

Median Range N

$\begin{array}{lll}0 & 0-0.3 & 15\end{array}$

$\begin{array}{lll}0 & 0-0.5 & 15\end{array}$

$\begin{array}{lll}0 & -0.5-0.3 \quad 15\end{array}$

$0.5 \quad 0.4-0.6 \quad 31$

$\begin{array}{lll}0 & 0-0.3 & 31\end{array}$

$0.5 \quad 0.1-0.6 \quad 31$

536 *Food sharing records do not exist for all 31 bats used for playbacks because some males

537

538

539 Table 2. Acoustic comparisons between playbacks or behavioural responses Acoustic distance by:

$\begin{array}{llll}\mathbf{R}^{\mathbf{2}} & \mathbf{d f} & \mathbf{F} & \mathbf{p} \\ 0.03 & 1,44 & 1.4 & 0.24 \\ 0.02 & 1,14 & 0.2 & 0.63 \\ 0.01 & 1,14 & 0.2 & 0.67 \\ 0.01 & 1,14 & 0.4 & 0.52 \\ 0.01 & 1,14 & 0.4 & 0.28\end{array}$

Test type (kinship vs. donor)

Time spent in test arm in donor test

Latency to enter test arm in donor test

Time spent in test arm in kinship test

Latency to enter test arm in kinship test

0.28 


$\begin{array}{lllll}\text { Absolute difference in mean amplitude by: } & & & & \\ \text { Test type (kinship vs. donor) } & <0.001 & 1,44 & 0.02 & 0.88 \\ \text { Time spent in test arm in donor test } & 0.01 & 1,14 & 0.2 & 0.69 \\ \text { Latency to enter test arm in donor test } & 0.002 & 1,14 & 0.03 & 0.86 \\ \text { Time spent in test arm in kinship test } & <0.001 & 1,14 & <0.01 & 0.98 \\ \text { Latency to enter test arm in kinship test } & 0.002 & 1,14 & 0.04 & 0.83\end{array}$


This study was made possible by collaboration with the Organization for Bat Conservation, with help from R. Mies, A. Felk, N. Siekert, J. Fabian, L. Dehn, and K. Vowles. The experimental maze was built by K. Sullenger. The work was supported by student research grants from the Animal Behavior Society and the American Society of Mammalogists, and a Doctoral Dissertation Improvement Grant from the National Science Foundation (\#1311336). We thank Brock Fenton, Ken Yasukawa, and an anonymous reviewer for suggestions that improved the manuscript. 
Figure 1

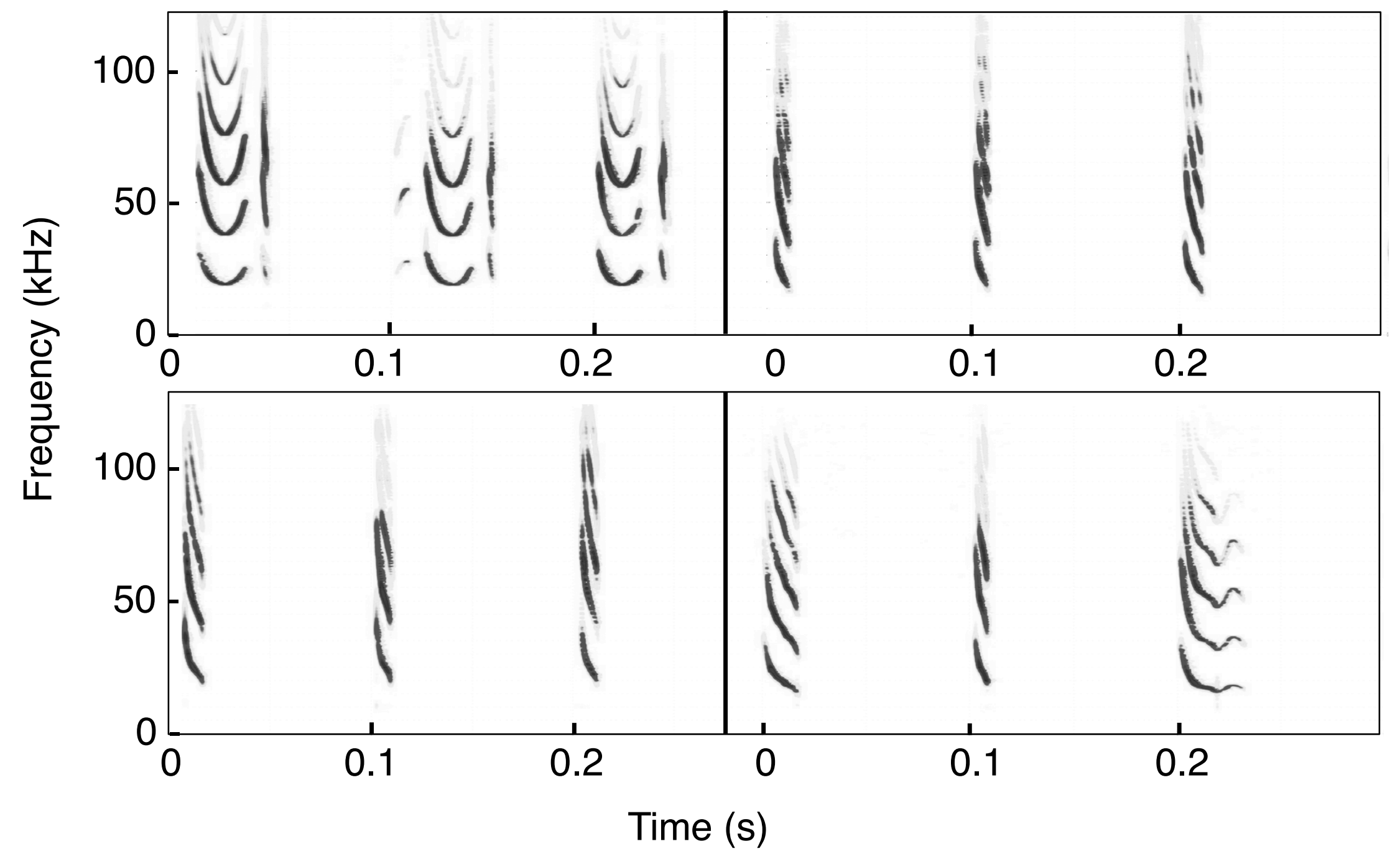




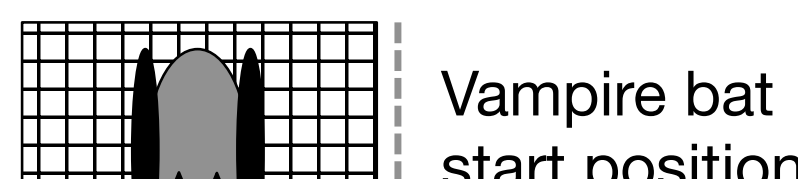

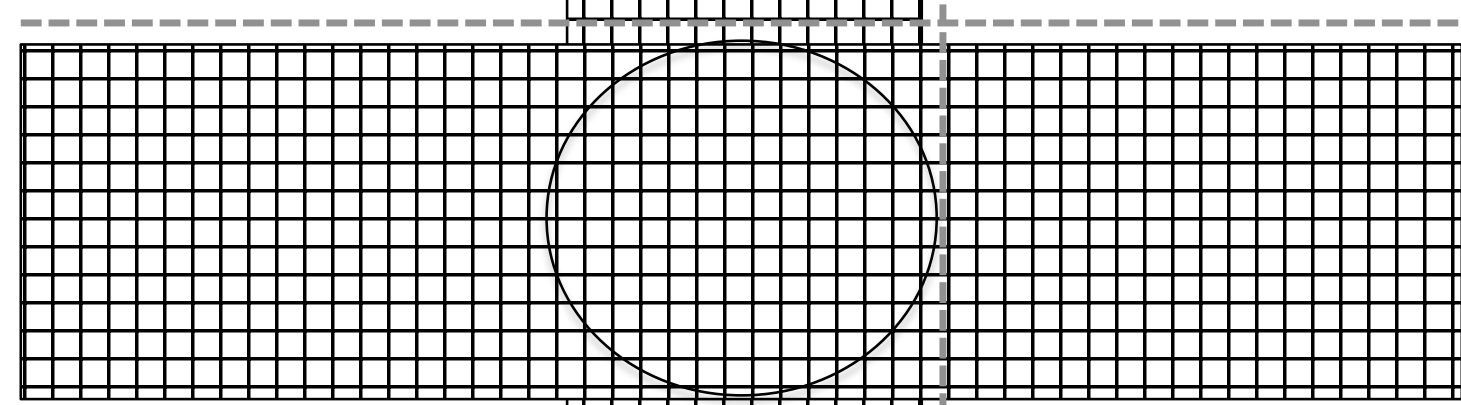

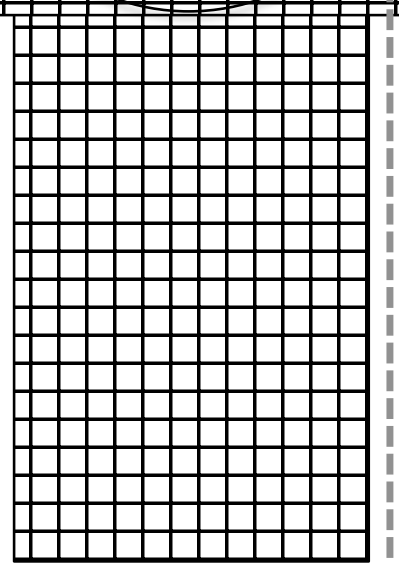

$76 \mathrm{~cm}$

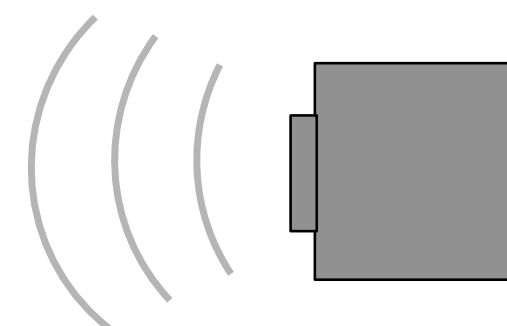

Ultrasonic speaker
Ultrasonic speaker
Video camcorder with infrared spotlight 


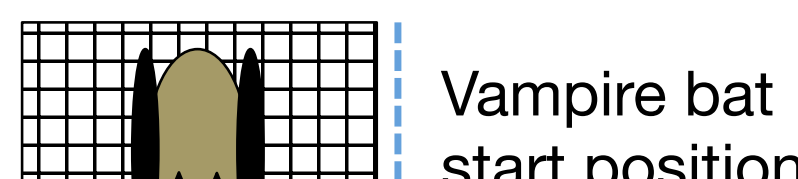

\section{}

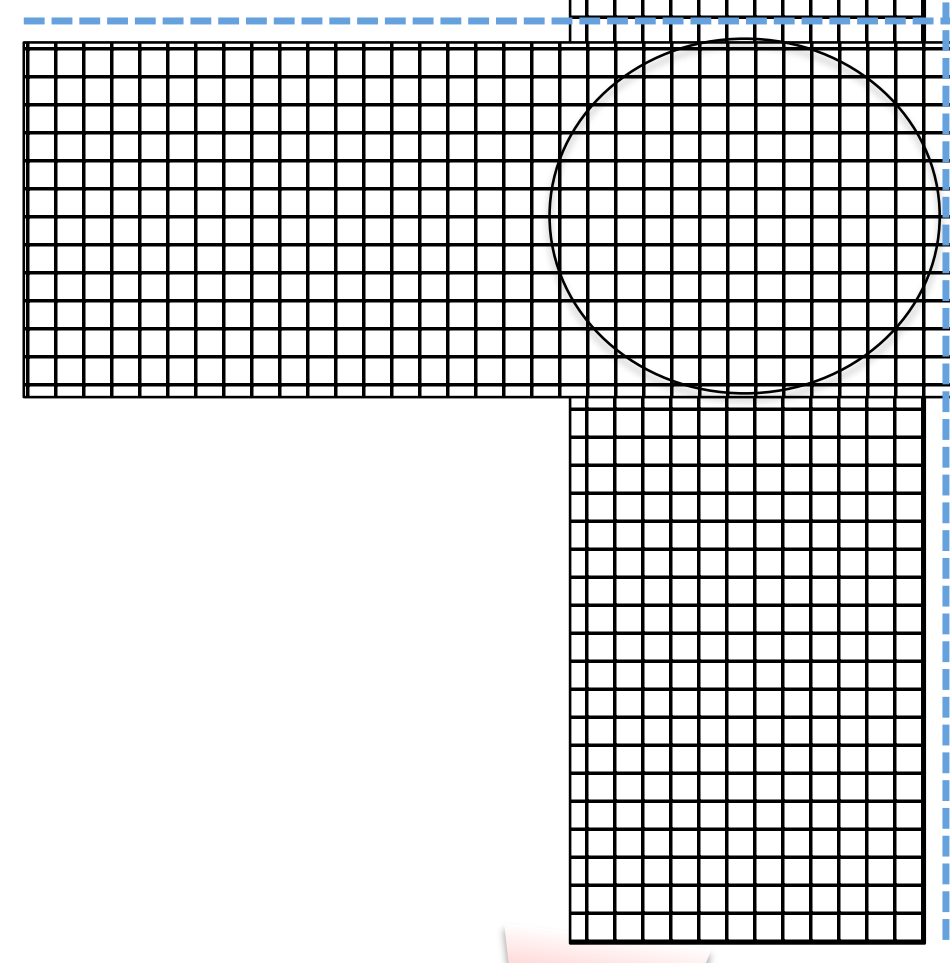

$76 \mathrm{~cm}$

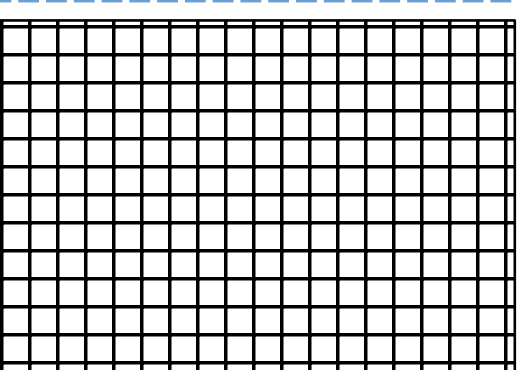

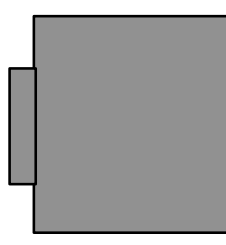

Ultrasonic speaker
Video camcorder with infrared spotlight 
(tag)

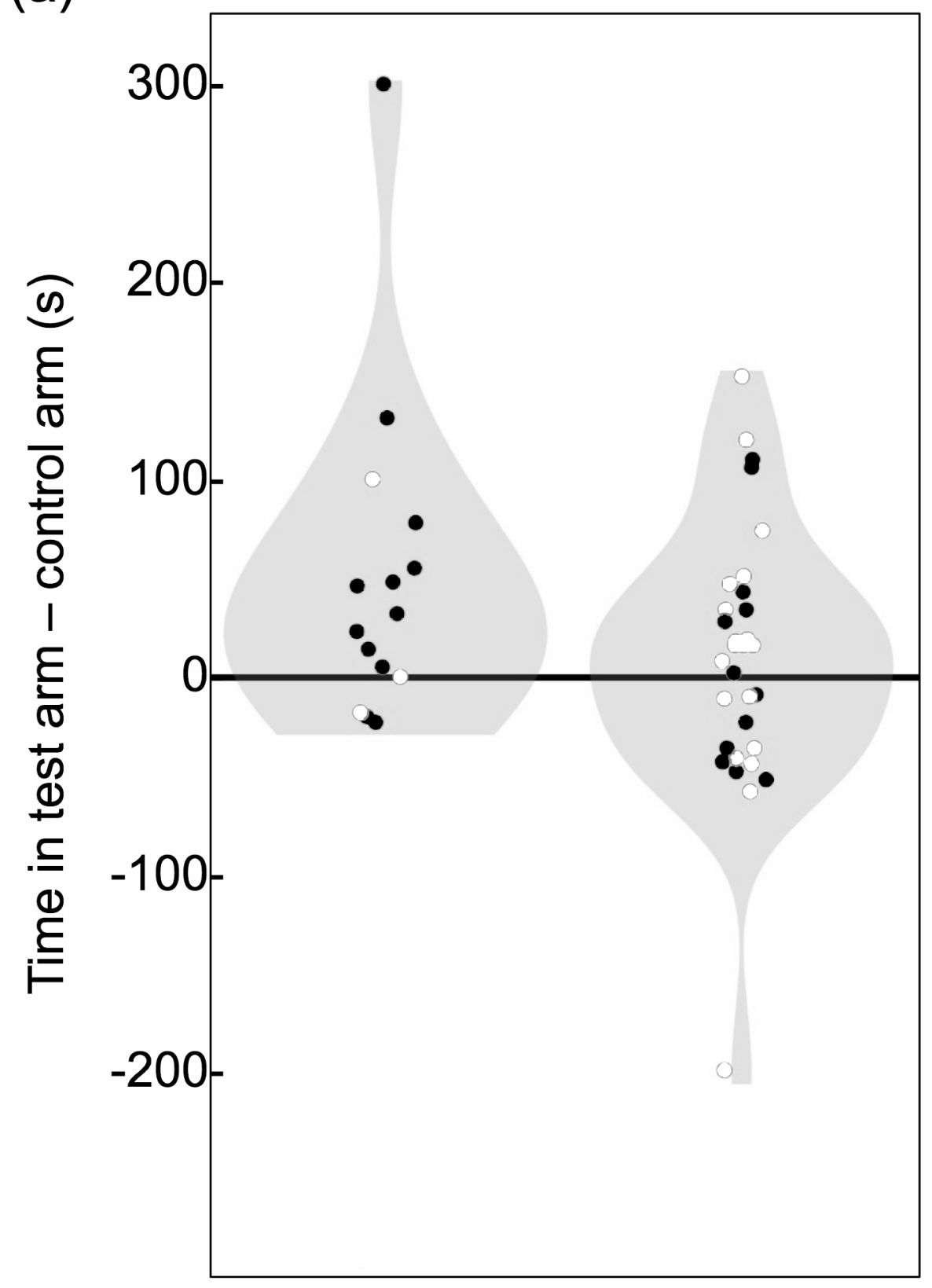

Donor test Kinship test (b)

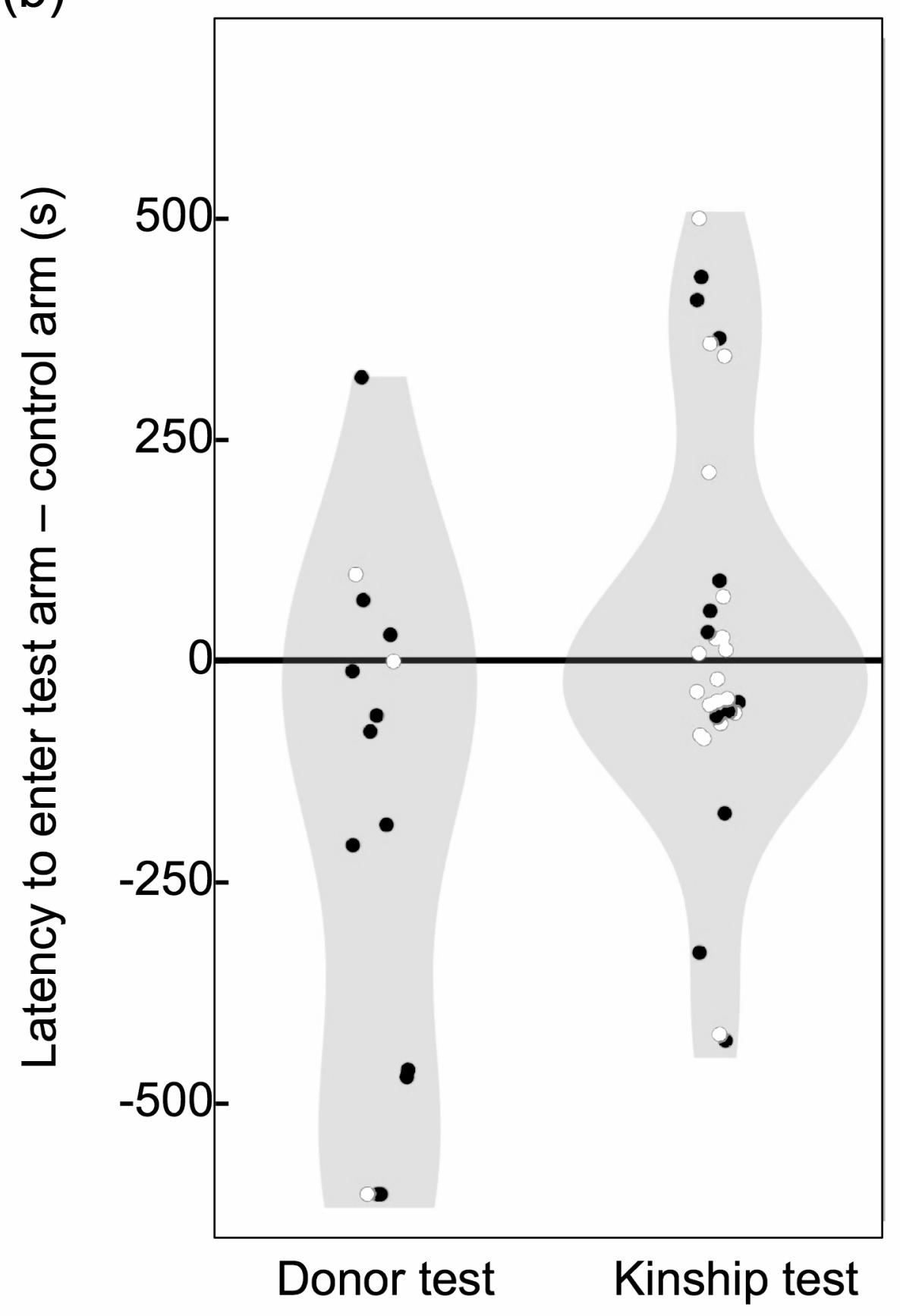




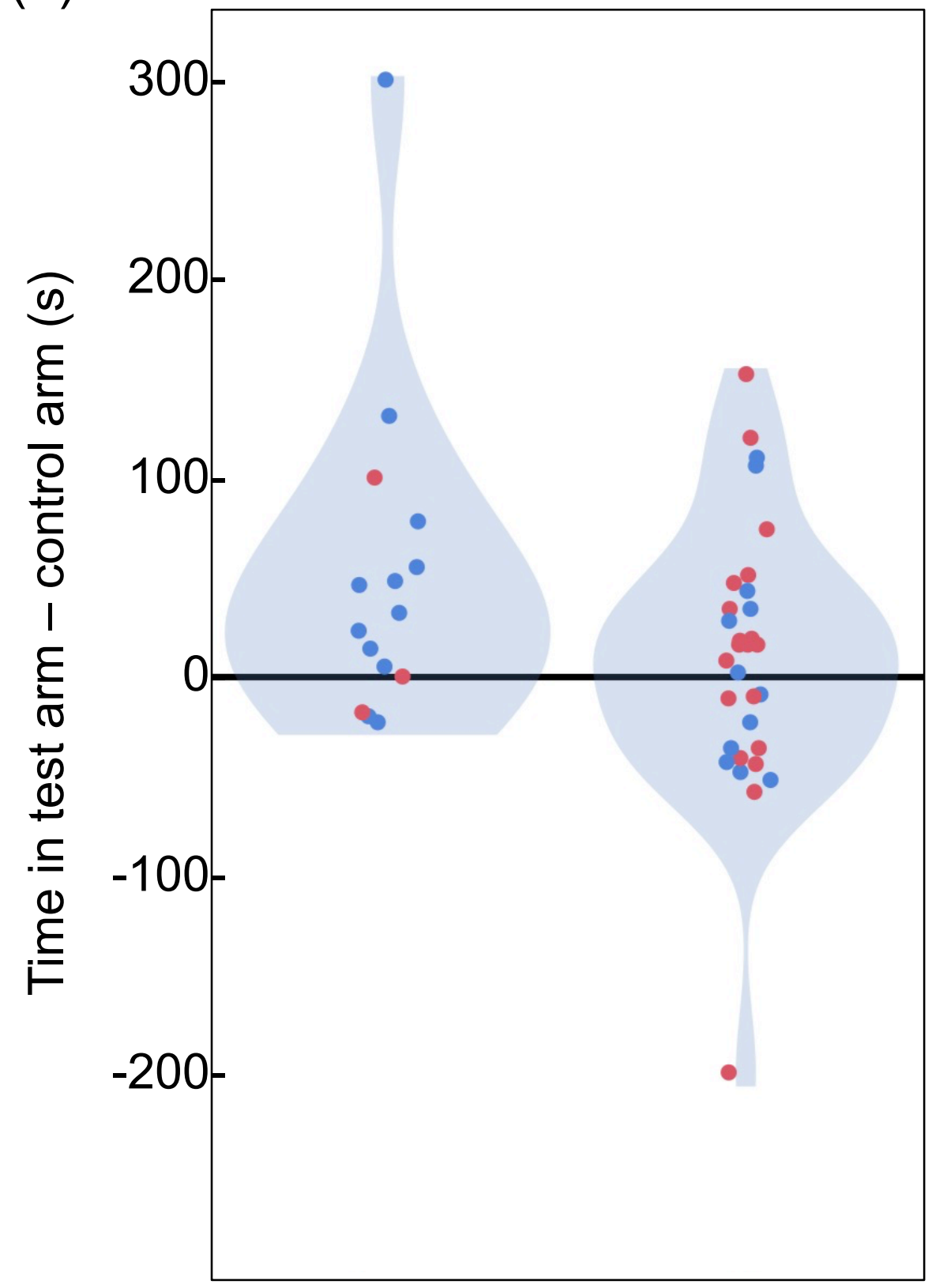

Donor test Kinship test

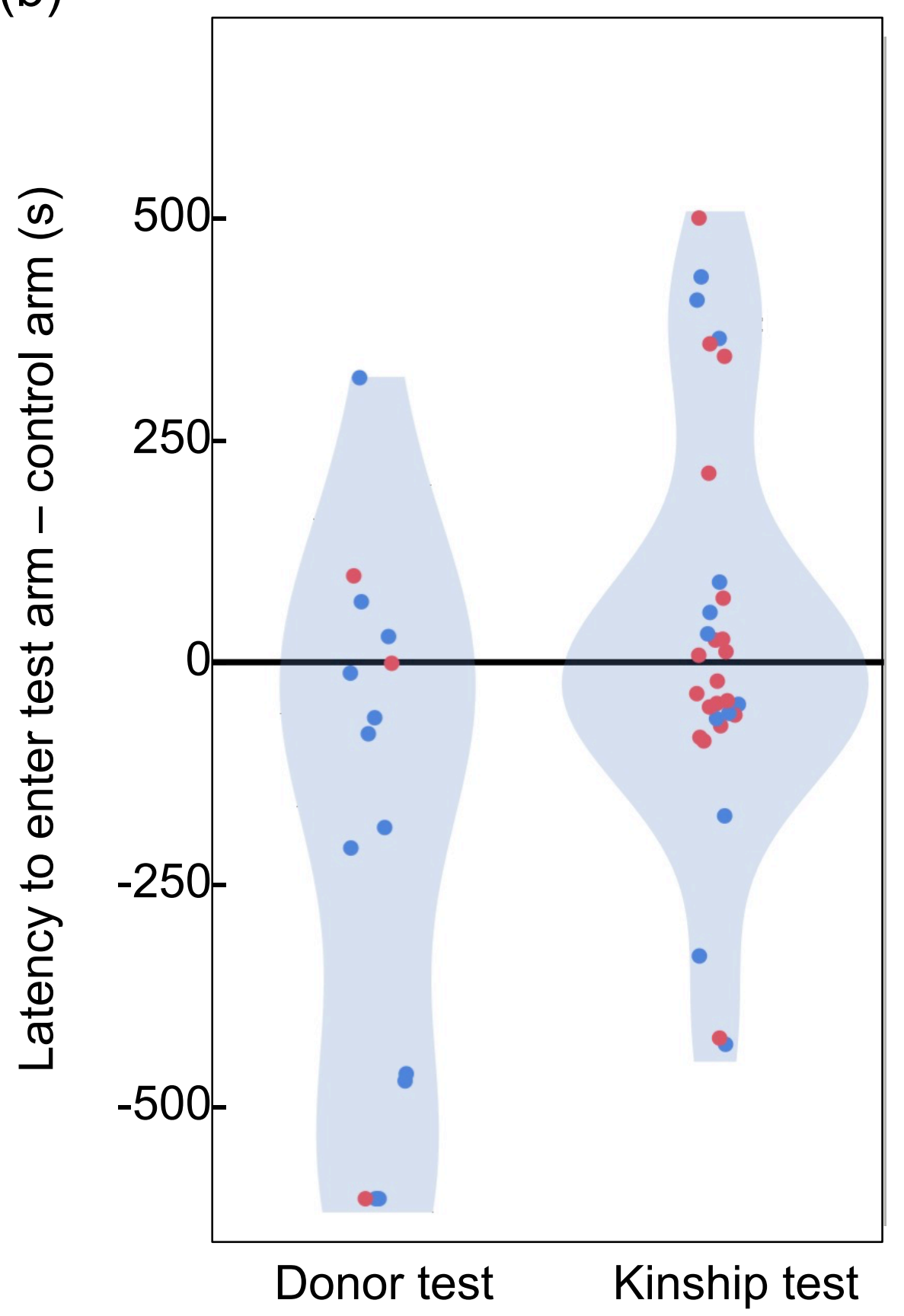

